\title{
On the Zeros of a Polynomial in a Given Domain
}

\author{
M. S. Pukhta \\ Division of Agricultural Statistics, Sher-e-Kashmir University of Agricultural Sciences \& Technology of Kashmir, Srinagar (191121), \\ India \\ *Corresponding Author: mspukhta_67@yahoo.co.in
}

Copyright (C) 2014Horizon Research Publishing All rights reserved.

Abstract In this paper we obtain results concerning the bound for the number of zeros for the polynomial $p(z)$ which generalizes well known result due to A.Ebadian ,M.Bidkham and M.Eshaghi Gordji [Number of zeros of a polynomial in a given domain, Tamkang Jour, of Mathematics, Vol 42, No.4,(2011), 531-536] and also improves upon some well-known results.

Keywords Polynomial, Zeros, Complex Number, Prescribed Region

\section{Introduction} that

$\operatorname{Let} p(z)=\sum_{j=o}^{n} a_{j} z^{j}$ be a polynomial of degree $\mathrm{n}$ such

$$
a_{n} \geq a_{n-1} \geq \cdots \geq a_{1} \geq a_{0}>0
$$

then according to a well known result of Enstrom and Kakeya, the polynomial $p(z)$ does not vanish in $|z|>$ 1.Concerning the number of zeros of the polynomial in the region $|z| \leq \frac{1}{2}$ the following result is due to Mohammad [1].

Theorem A. Let $p(z)=\sum_{j=o}^{n} a_{j} z^{j}$ be a polynomial of degree $\mathrm{n}$ such that

$$
a_{n} \geq a_{n-1} \geq \cdots \geq a_{1} \geq a_{0}>0
$$

then the number of zeros of $p(z)$ in $|z| \leq \frac{1}{2}$ does not exceed

$$
1+\frac{1}{\log 2} \log \frac{a_{n}}{a_{0}}
$$

Bidkham and Dewan [2] generalized the Theorem A for different class of polynomials and proved the following.

Theorem B.Let $p(z)=\sum_{j=o}^{n} a_{j} z^{j}$ be a polynomial of degree $\mathrm{n}$ such that

$$
a_{n} \leq a_{n-1} \leq \cdots \leq a_{k+1} \leq a_{k} \geq a_{k-1} \geq \cdots \geq a_{0}
$$

for some $k, 0 \leq k \leq n$, then the number of zeros of $p(z)$ in $|z| \leq \frac{1}{2}$ does not exceed

$$
\frac{1}{\log 2}\left\{\log \frac{\left|a_{n}\right|+\left|a_{0}\right|-a_{n}-a_{0}+2 a_{k}}{\left|a_{0}\right|}\right\}
$$

Theorem C. Let $p(z)=\sum_{j=o}^{n} a_{j} z^{j}$ be a polynomial of degree $\mathrm{n}$ with complex coefficients .If for some real $\beta,\left|\arg \mathrm{a}_{\mathrm{j}}-\beta\right| \leq \alpha \leq \frac{\pi}{2}, 0 \leq j \leq n$

and for some $0<t \leq 1$,

$$
\begin{gathered}
\left|a_{0}\right| \leq t\left|a_{1}\right| \leq \cdots \leq t^{k}\left|a_{k}\right| \geq t^{k+1}\left|a_{k+1}\right| \geq \cdots \geq t^{n}\left|a_{n}\right|, \\
o \leq k \leq n
\end{gathered}
$$

then the number of zeros of $p(z)$ in $|z| \leq \frac{1}{2}$ does not exceed

$$
\frac{1}{\log 2}\left\{\log \frac{2 \mathrm{t}^{\mathrm{k}+1}\left|\mathrm{a}_{\mathrm{k}}\right| \cos \alpha+2 \mathrm{t} \sin \alpha \sum_{\mathrm{j}=0}^{\mathrm{n}} \mathrm{t}^{\mathrm{t}}\left|\mathrm{a}_{\mathrm{j}}\right|-\mathrm{t}^{\mathrm{n}+1}\left|\mathrm{a}_{\mathrm{n}}\right|(\cos \alpha+\sin \alpha-1)}{\mathrm{t}\left|\mathrm{a}_{0}\right|}\right\}
$$

A.Ebaddian,M.Bidkham and M.Eshaghi Gordji [3] generalizes Theorem $\mathrm{C}$ and proved the following results.

Theorem D. Let $p(z)=\sum_{j=o}^{n} a_{j} z^{j}$ be a polynomial of degree $n$ such that

$$
\mathrm{a}_{\mathrm{n}} \leq \mathrm{a}_{\mathrm{n}-1} \leq \cdots \leq \mathrm{a}_{\mathrm{k}+1} \leq \mathrm{a}_{\mathrm{k}} \geq \mathrm{a}_{\mathrm{k}-1} \geq \cdots \geq \mathrm{a}_{0}
$$

for some $k, 0 \leq k \leq n$, then the number of zeros of $p(z)$ in $|z| \leq \frac{R}{2}, R>0$, does not exceed

$\frac{1}{\log 2}\left\{\log \frac{\left|a_{n}\right| R^{n+1}+\left|a_{0}\right|+R^{k}\left(a_{k}-a_{0}\right)+R^{n}\left(a_{k}-a_{n}\right)}{\left|a_{0}\right|}\right\}$

for $R \geq 1$

$\frac{1}{\log 2}\left\{\log \frac{\left|a_{n}\right| R^{n+1}+\left|a_{0}\right|+R\left(a_{k}-a_{0}\right)+R^{n}\left(a_{k}-a_{n}\right)}{\left|a_{0}\right|}\right\}$

For $R \leq 1$

Theorem E. Let $p(z)=\sum_{j=o}^{n} a_{j} z^{j}$ be a polynomial of degree $\mathrm{n}$ with complex coefficients .If for some real $\beta,\left|\arg \mathrm{a}_{\mathrm{j}}-\beta\right| \leq \alpha \leq \frac{\pi}{2}, 0 \leq j \leq n$ and for some $R>0$,

$$
\begin{gathered}
\left|a_{0}\right| \leq R\left|a_{1}\right| \leq \cdots \leq R^{k}\left|a_{k}\right| \geq R^{k+1}\left|a_{k+1}\right| \geq \cdots \geq \\
R^{n}\left|a_{n}\right|, o \leq k \leq n
\end{gathered}
$$

then the number of zeros of $p(z)$ in $|\mathrm{z}| \leq \frac{\mathrm{R}}{2}, \mathrm{R}>0$ does not exceed

$\frac{1}{\log 2}\left\{\log \frac{2 \mathrm{R}^{\mathrm{k}+1}\left|\mathrm{a}_{\mathrm{k}}\right| \cos \alpha+2 \mathrm{R} \sin \alpha \sum_{\mathrm{j}=0}^{\mathrm{n}} \mathrm{R}^{\mathrm{j}}\left|\mathrm{a}_{\mathrm{j}}\right|-\mathrm{R}^{\mathrm{n}+1}\left|\mathrm{a}_{\mathrm{n}}\right|(\cos \alpha+\sin \alpha-1)}{\mathrm{R}\left|\mathrm{a}_{0}\right|}\right\}$

In this paper we improve Theorem D and Theorem $\mathrm{E}$ for polynomials with real and complex coefficients. More 
precisely we prove

Theorem 1 .Let $p(z)=\sum_{j=o}^{n} a_{j} z^{j}$ be a polynomial of degree $n$ such that

$$
\mathrm{a}_{\mathrm{n}} \leq \mathrm{a}_{\mathrm{n}-1} \leq \cdots \leq \mathrm{a}_{\mathrm{k}+1} \leq \mathrm{a}_{\mathrm{k}} \geq \mathrm{a}_{\mathrm{k}-1} \geq \cdots \geq \mathrm{a}_{0}
$$

for some $k, 0 \leq k \leq n$, then the number of zeros of $p(z)$ in $|\mathrm{z}| \leq \mathrm{R} \delta, \mathrm{R}>0$, and $0<\delta<1$ does not exceed

$\frac{1}{\log \frac{1}{\delta}}\left\{\log \frac{\left|a_{n}\right| R^{n+1}+\left|a_{0}\right|+R^{k}\left(a_{k}-a_{0}\right)+R^{n}\left(a_{k}-a_{n}\right)}{\left|a_{0}\right|}\right\}$ for $R \geq 1$

and

$$
\frac{1}{\log \frac{1}{\delta}}\left\{\log \frac{\left|a_{n}\right| R^{n+1}+\left|a_{0}\right|+R\left(a_{k}-a_{0}\right)+R^{n}\left(a_{k}-a_{n}\right)}{\left|a_{0}\right|}\right\}
$$$$
\text { for } R \leq 1
$$

Remark 1.1. If we choose $=\frac{1}{2}$, Theorem 1.reduces to Theorem D.

Remark 1.2. If we choose $=1, \delta=\frac{1}{2}$. Theorem 1 reduces to Theorem $\mathrm{B}$.

Theorem 2. Let $p(z)=\sum_{j=o}^{n} a_{j} z^{j}$ be a polynomial of degree $\mathrm{n}$ with complex coefficients. If for some real $\beta$, $\mid$ arg $\mathrm{a}_{\mathrm{j}}-\beta \mid \leq \alpha \leq \frac{\pi}{2}, 0 \leq j \leq n$ and for some $R>0$,

$$
\begin{aligned}
\left|a_{0}\right| \leq R\left|a_{1}\right| \leq \cdots \leq R^{k}\left|a_{k}\right| & \geq R^{k+1}\left|a_{k+1}\right| \geq \cdots \geq \\
R^{n}\left|a_{n}\right| \quad, \quad o & \leq k \leq n
\end{aligned}
$$

then the number of zeros of $p(z)$ in $|\mathrm{z}| \leq \mathrm{R} \delta, \mathrm{R}>$ $0,0<\delta<1$ does not exceed

$\frac{1}{\log \frac{1}{\delta}}\left\{\log \frac{2 \mathrm{R}^{\mathrm{k}+1}\left|\mathrm{a}_{\mathrm{k}}\right| \cos \alpha+2 \mathrm{R} \sin \alpha \sum_{\mathrm{j}=0}^{\mathrm{n}} \mathrm{R}^{\mathrm{j}}\left|\mathrm{a}_{\mathrm{j}}\right|-\mathrm{R}^{\mathrm{n}+1}\left|\mathrm{a}_{\mathrm{n}}\right|(\cos \alpha+\sin \alpha-1)}{\mathrm{R}\left|\mathrm{a}_{0}\right|}\right\}$

Remark 2.1.If $=\frac{1}{2}$, Theorem 2 reduces to Theorem E.

Remark 2.2. For $R=1, k=1, \delta=\frac{1}{2}$ and $\alpha=\beta=0$. Theorem 2 reduces to Theorem A.

\section{Lemma}

For the proof of the theorems, we need the following lemmas.

Lemma 2.1. If $f(z)$ is regular, $f(0 \neq 0)$ and $f(z) \leq M$ in $|z| \leq 1$, then see ([4],pp 171) the number of zeros of $f(z)$ in $|z| \leq \delta, 0<\delta<1$ does not exceed

$$
\frac{1}{\log \frac{1}{\delta}} \log \frac{M}{|f(0)|}
$$

Lemma 2.2. Let $p(z)=\sum_{j=o}^{n} a_{j} z^{j}$ be a polynomial of degree $\mathrm{n}$ with such that $\left|\arg \mathrm{a}_{\mathrm{j}}-\beta\right| \leq \alpha \leq \frac{\pi}{2}, 0 \leq j \leq n$ for some real $\beta$, then for some $t>0$,

$$
\begin{gathered}
\left|t a_{j}-a_{j-1}\right| \leq\left(t\left|a_{j}\right|-\left|a_{j-1}\right|\right) \cos \alpha \\
+\left(t\left|a_{j}\right|+\left|a_{j-1}\right|\right) \sin \alpha
\end{gathered}
$$

The above lemma is due to Govil [5].

\section{Proof of the Theorems}

Proof of Theorem 1. Consider the polynomial

$$
\begin{gathered}
g(z)=(1-z) p(z) \\
=-a_{n} z^{n}+a_{0}+\sum_{j=1}^{n}\left(a_{j}-a_{j-1}\right) z^{j}
\end{gathered}
$$

For $|z| \leq R$, we have

$$
\begin{aligned}
& |g(z)| \leq\left|a_{n}\right| R^{n}+\left|a_{0}\right| \\
& +\sum_{j=1}^{k}\left(a_{j}-a_{j-1}\right) R^{j} \\
& +\sum_{j=k+1}^{n}\left(a_{j-1}-a_{j}\right) R^{j}
\end{aligned}
$$

Which gives

$$
\begin{gathered}
|g(z)| \leq\left|a_{n}\right| R^{n+1}+\left|a_{0}\right|+R^{k}\left(a_{k}-a_{0}\right)+R^{n}\left(a_{k}-a_{n}\right) \\
\text { for } R \geq 1
\end{gathered}
$$

and

$$
\begin{gathered}
|g(z)| \leq\left|a_{n}\right| R^{n+1}+\left|a_{0}\right|+R\left(a_{k}-a_{0}\right)+R^{n}\left(a_{k}-a_{n}\right) \\
\text { for } R \leq 1
\end{gathered}
$$

Which further imply

$$
\begin{gathered}
\left|\frac{g(z)}{g(0)}\right| \leq \frac{\left|a_{n}\right| R^{n+1}+\left|a_{0}\right|+R^{k}\left(a_{k}-a_{0}\right)+R^{n}\left(a_{k}-a_{n}\right)}{\left|a_{0}\right|} \\
\text { for } R \geq 1
\end{gathered}
$$

And

$$
\begin{gathered}
\left|\frac{g(z)}{g(0)}\right| \leq \frac{\left|a_{n}\right| R^{n+1}+\left|a_{0}\right|+R\left(a_{k}-a_{0}\right)+R^{k}\left(a_{k}-a_{n}\right)}{\left|a_{0}\right|} \\
\text { for } R \leq 1
\end{gathered}
$$

Applying Lemma 2.1 to $(z)$, we get the number of zeros of $g(z)$ in $|z| \leq R \delta$, does not exceed

$\frac{1}{\log \frac{1}{\delta}}\left\{\log \frac{\left|a_{n}\right| R^{n+1}+\left|a_{0}\right|+R^{k}\left(a_{k}-a_{0}\right)+R^{n}\left(a_{k}-a_{n}\right)}{\left|a_{0}\right|}\right\}$

for $R \geq 1$

$$
\begin{gathered}
\text { and } \\
\log \frac{1}{\delta}
\end{gathered}\left\{\log \frac{\left|a_{n}\right| R^{n+1}+\left|a_{0}\right|+R\left(a_{k}-a_{0}\right)+R^{k}\left(a_{k}-a_{n}\right)}{\left|a_{0}\right|}\right\}
$$

As the number of zeros of $p(z)$ in $|z| \leq R \delta$ does not exceed the number of zeros of $g(z)$ in $|z| \leq R \delta$, the theorem follows.

\section{Proof of Theorem 2.}

Consider

$$
F(z)=(R-z) p(z)
$$




$$
=-a_{n} z^{n+1}+R a_{0}+\sum_{j=1}^{n}\left(R a_{j}-a_{j-1}\right) z^{j}
$$

For $|z| \leq R$, we have

$$
\begin{gathered}
|F(z)| \leq\left|a_{n}\right| R^{n+1}+R\left|a_{0}\right| \\
+\sum_{j=1}^{n}\left(R\left|a_{j}\right|-\left|a_{j-1}\right|\right) R^{j} \cos \alpha \\
+\sum_{j=1}^{n}\left(R\left|a_{j}\right|+\left|a_{j-1}\right|\right) R^{j} \sin \alpha \\
=\left|a_{n}\right| R^{n+1}+R\left|a_{0}\right| \\
+\sum_{j=1}^{k}\left(R\left|a_{j}\right|-\left|a_{j-1}\right|\right) R^{j} \cos \alpha \\
+\sum_{j=k+1}^{n}\left(\left|a_{j-1}\right|+R\left|a_{j}\right|\right) R^{j} \cos \alpha \\
+\sum_{j=1}^{n}\left(R\left|a_{j}\right|+\left|a_{j-1}\right|\right) R^{j} \sin \alpha \\
=2 R^{k+1}\left|a_{k}\right| \cos \alpha \\
+2 R \sin \alpha \sum_{j=0}^{n} R^{j}\left|a_{j}\right| \\
-R\left|a_{0}\right|(\cos \alpha+\sin \alpha-1) \\
-R^{n+1}\left|a_{n}\right|(\cos \alpha+\sin \alpha-1)
\end{gathered}
$$

$$
\begin{gathered}
\leq 2 R^{k+1}\left|a_{k}\right| \cos \alpha \\
+2 R \sin \alpha \sum_{j=0}^{n} R^{j}\left|a_{j}\right| \\
-R^{n+1}\left|a_{n}\right|(\cos \alpha+\sin \alpha-1)
\end{gathered}
$$

Further proceeding on the same lines of Theorem 1, the proof of Theorem 2 can be completed.

\section{REFERENCES}

[1] Q. G. Mohammad, on the zeros of the polynomials, American Mathematically Monthly, vol.72, No. 6, (1965), 631-633.

[2] M. Bidjham and K.K.Dewan, on the zeros of a polynomial, Numerical Methods and approximation Theory 111, NIS (1987), 121-128.

[3] A.Ebadian, M.Bidkham and M.Eshaghi Gordji Number of zeros of a polynomial in a given domain, Tamkang Jour, of Mathematics, Vol 42, No.4, (2011), 531-536.

[4] E.C.Titchmarsh, The Theory of functions, 2nd ed, Oxford University Press, London, 1939.

[5] N.K.Govil and Q.I.Rehman, On the EnstromKakeya Theorem Tohoku Math .J. 20, (1968), 126-136. 